of possible causes before intubation. In some patients, postoperative intubation is not required and it is possible to follow a more conservative plan of therapy. In others intubation is not a benign procedure and carries some risk, and necessitates an intensive care unit admission.

Again, I would like to thank $D r$. Tousignant for bringing his previous article to our attention.

George A. Arndt MD

Madison, Wisconsin, USA

\section{Incorrect analysis of data leads to incorrect conclusions}

To the Editor:

Recently, Aye et al. compared cardiac output (CO) estimation by visual inspection $v s$ thermodilution during cardiac surgery. ${ }^{1}$ They utilized Bland and Altman analysis ${ }^{2}$ appropriately, but overlooked the first step of the method, thus invalidating their interpretation of the results.

This statistical approach involves two stages. ${ }^{2,3}$ First, a decision must be made as to how large a difference between the two methods is permissible while still supporting the conclusion that the two methods are interchangeable. This decision is often arbitrary, based on clinical judgment. Second, results are plotted as described in the article. The two methods are judged to interchangeable if the limits of agreement $( \pm 2$ SD of the mean difference between the two methods) do not exceed the chosen acceptable difference (the shaded area in the Figure).

Given the normal range of $\mathrm{CO}$ and the inherent variability of thermodilution $\mathrm{CO}$ determination, we determined a difference $\leq 1 \mathrm{~L} \cdot \mathrm{min}^{-1}$ (or $\pm 0.5 \mathrm{~L} \cdot \mathrm{min}^{-1}$ ) between the two methods would be clinically acceptable. Based on the data presented in the article, we calculated the anaesthetist's evaluation ranged from $2.45 \mathrm{~L} \cdot \mathrm{min}^{-1}$ below to $3.63 \mathrm{~L} \cdot \mathrm{min}^{-1}$ above thermodilution $\mathrm{CO}$ measurement, far exceeding the chosen acceptable difference of $\pm \mathrm{L} \cdot \mathrm{min}^{-1}$. Only 13 (approximately) of the 35 data points $(37 \%)$ are within the chosen acceptable difference.

Whatever our beliefs may be regarding the value of pulmonary artery catheterization to improve patient outcome, we must conclude that visual inspection and thermodilution are not interchangeable methods to determine $\mathrm{CO}$ during cardiac surgery.

Jean-François Hardy MD

Sylvain Bélisle $M D$

Normand Gravel MD

Montréal, Québec

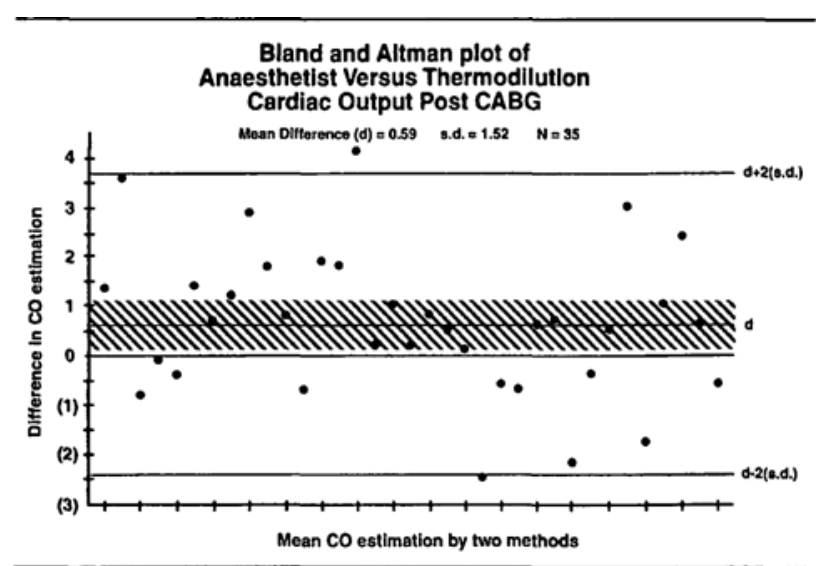

FIGURE Bland and Altman plot reproduced from the article by Aye et al. comparing the anaesthetists' subjective estimates $p s$ thermodilution determination for $\mathrm{CO} .^{1}$ We added the shaded area representing the difference chosen to be acceptable while still supporting the conclusion that the two methods are interchangeable $\left(0.5 \mathrm{~L} \cdot \mathrm{min}^{-1}\right.$ above and below the mean difference i.e., biasof $\left.0.59 \mathrm{~L} \cdot \mathrm{min}^{-1}\right)$. The limits of agreement $( \pm 2 \mathrm{SD})$ exceed the shaded area, indicating the two methods are not interchangeable.

\section{REFEREN CES}

I Aye $T$, Milne B, Ballantyne $M$. Cardiac output estimation by visual inspection vs thermodilution during cardiac surgery. Can J Anacsth 1997; 44: 126-30.

2 Bland JM, Altman DG. Statistical methods for assessing agreement between two methods of clinical measurement. Lancet 1986; 1: 307-10.

3 LaMantia KR, O'Connor T, Barash PG. Comparing methods of measurement: an alternative approach (Editorial). Anesthesiology 1990; 72: 781-3.

\section{REPLY}

"If error is corrected whenever it is recognized as such, the path of error is the path of truth.

(HANs ReICHENBACH)

We thank Hardy, Belisle and Gravel for their insightful interest in our paper. ${ }^{1}$ We did use the Bland-Altman ${ }^{2,3}$ statistical approach to test the comparability of the surgeons' visual inspection estimates vs the thermodilution measurement and again for the anaesthetists' visual inspection estimates vs thermodilution. A correlation coefficient would not be appropriate for this type of analysis as this correlation statistic is not a measure of agreement between two measures, but of association.

Thermodilution is considered to be the gold standard for measuring cardiac output. Nevertheless, cardiac output measurements after cardiopulmonary bypass may have errors of 15-50\% ${ }^{4}$ which is greater than the inherent variability quoted by Hardy et al.: i.e., botb thermodilution and visual inspection are "noisy" measures. 